\title{
Characterization of Structural Variations in the Peptidoglycan of Vancomycin-Susceptible Enterococcus faecium: Understanding Glycopeptide-Antibiotic Binding Sites Using Mass Spectrometry
}

\author{
Gary J. Patti, Jiawei Chen, Jacob Schaefer, and Michael L. Gross \\ Department of Chemistry, Washington University, St. Louis, Missouri, USA
}

Enterococcus faecium, an opportunistic pathogen that causes a significant number of hospitalacquired infections each year, presents a serious clinical challenge because an increasing number of infections are resistant to the so-called antibiotic of last resort, vancomycin. Vancomycin and other new glycopeptide derivatives target the bacterial cell wall, thereby perturbing its biosynthesis. To help determine the modes of action of glycopeptide antibiotics, we have developed a bottom-up mass spectrometry approach complemented by solid-state nuclear magnetic resonance (NMR) to elucidate important structural characteristics of vancomycinsusceptible E. faecium peptidoglycan. Using accurate-mass measurements and integrating ion-current chromatographic peaks of digested peptidoglycan, we identified individual muropeptide species and approximated the relative amount of each. Even though the organism investigated is susceptible to vancomycin, only $3 \%$ of the digested peptidoglycan has the well-known D-Ala-D-Ala vancomycin-binding site. The data are consistent with a previously proposed template model of cell-wall biosynthesis where D-Ala-D-Ala stems that are not cross-linked are cleaved in mature peptidoglycan. Additionally, our mass-spectrometry approach allowed differentiation and quantification of muropeptide species seen as unresolved chromatographic peaks. Our method provides an estimate of the extent of muropeptides containing $\mathrm{O}$-acetylation, amidation, hydroxylation, and the number of species forming cyclic imides. The varieties of muropeptides on which the modifications are detected suggest that significant processing occurs in mature peptidoglycan where several enzymes are active in editing cell-wall structure. (J Am Soc Mass Spectrom 2008, 19, 1467-1475) (C) 2008 American Society for Mass Spectrometry

$\mathrm{B}$ acterial peptidoglycan is an attractive drug target because it has unique chemistry, and its integrity is crucial to protect cells from internal osmotic pressure. Vancomycin, a glycopeptide antibiotic targeting the peptidoglycan, has become increasingly important given its effectiveness against multiple drugresistant bacteria. Alarmingly, some pathogens have developed resistance to even vancomycin, the so-called drug of last resort, leaving clinicians with limited treatment options. Among the most prevalent of these feared pathogens are vancomycin-resistant enterococci (VRE).

VRE infections, particularly Enterococcus faecium, have emerged as life-threatening pathogens in clinical settings worldwide. Since the first vancomycin-resistant E. faecium isolate was identified in 1986 [1], the number

Address reprint requests to Professor M. L. Gross, Department of Chemistry, Washington University, One Brookings Drive, Campus Box 1134, St. Louis, MO 63130, USA. E-mail: mgross@wustl.edu

* These two are joint first authors. of VRE infections has increased dramatically, causing high mortality rates around the world. In some hospitals, over $90 \%$ of E. faecium isolates are vancomycin resistant [2]. This presents a significant clinical challenge and manifests the urgency to find new therapeutic treatment possibilities.

Vancomycin inhibits peptidoglycan biosynthesis of the bacterial cell wall by forming complexes with the D-Ala-D-Ala carboxyl termini of peptidoglycan precursors outside the cytoplasmic membrane [3]. Enterococcal resistance is generally thought to involve the modification of D-Ala-D-Ala binding sites to D-Ala-D-Lac [4]. This change replaces a hydrogen bond between the nitrogen of the terminal D-Ala and the oxygen of vancomycin with an oxygen-oxygen repulsive interaction. The modified vancomycin-peptidoglycan stem complex has an in vitro binding constant over 1000 -fold less than that involving the D-Ala-D-Ala stem [5].

Recently, it was suggested that potent vancomycinlike glycopeptides, effective against VRE, recognize other peptidoglycan structural motifs in addition to 
D-Ala-D-Ala [6, 7]. To understand the modes of action of these new glycopeptide analogues, as well as the action of vancomycin itself, atomic-level detail of the enterococcal peptidoglycan is required. To accomplish this objective, we have used bottom-up mass spectrometry to investigate the structure of vancomycinsusceptible E. faecium peptidoglycan. In particular, we are interested in the detailed structure of E. faecium peptidoglycan, as these structural details may be important to understanding cell-wall architecture and drug resistance.

Previously, mass spectrometric interrogations of enterococcal peptidoglycan did not address detailed muropeptide structural variations $[8,9]$. Those efforts, as well as other mass spectrometric analyses of bacterial peptidoglycan, relied on off-line MS identification [10-14] of HPLC peaks quantified by ultraviolet absorbance $[15,16]$. The strategy has proven to be powerful in characterizing the bacterial cell walls of many different organisms [14]. Given the complexity of the biological system and difficulty of complete chromatographic separation, however, each HPLC peak may represent an indistinguishable mixture of muropeptides, some of which may not be assigned.

The approach we report here is to use liquid chromatography-mass spectrometry (LC/MS) coupled with accurate-mass measurements of digested peptidoglycan to identify individual muropeptide species and, by integrating selected ion-current chromatographic peaks, estimate the relative amount of each. We verified the accurate-mass identifications with MS/MS and stableisotope labeling experiments. After specifically enriching amino acids in the peptidoglycan stem with stable-isotope labels, we examined unique muropeptide mass shifts to check our assignments. The sensitivity of our method allowed us to assign nearly $90 \%$ of the peaks in those mass spectra occurring within the range of expected muropeptide retention times.

\section{Experimental}

\section{Sample Preparation}

Starting cultures of E. faecium (ATTC 49624) were prepared by inoculating brain-heart infusion media with a single colony. Cultures were incubated overnight at $37^{\circ} \mathrm{C}$, but not aerated. Research samples were prepared by inoculating either brain-heart infusion or sterile enterococcal standard media (ESM) with the overnight starter cultures ( $1 \%$ final volume).

ESM was prepared as described before [17]. The $\mathrm{pH}$ of ESM was adjusted to 7.0 before sterile filtration. Natural abundance amino acids in ESM were replaced by stable ${ }^{13} \mathrm{C}$ and ${ }^{15} \mathrm{~N}$ isotope-enriched amino acids to incorporate specific labels into the samples for stableisotope labeling experiments. All samples enriched with D- $\left[1-{ }^{13} \mathrm{C}\right]$ alanine were treated with $5 \mu \mathrm{g} / \mathrm{mL}$ of alaphosphin, an alanine racemase inhibitor, every $1.5 \mathrm{~h}$ of growth to prevent scrambling of the isotopic label from D-Ala to L-Ala [17].

Cells were harvested at the end of log phase, when the absorbance at $660 \mathrm{~nm}$ became $\sim 1.0$, by centrifugation at $10,000 \times g$ for $25 \mathrm{~min}$ at $4{ }^{\circ} \mathrm{C}$. Pellets were rinsed with $40 \mathrm{mM}$ triethanolamine hydrochloride $(\mathrm{pH} 7.0)$ three times followed by three rinses with $\mathrm{H}_{2} \mathrm{O}$, centrifuging after each rinse. Cells were then resuspended in $\mathrm{H}_{2} \mathrm{O}$, frozen, and lyophilized. Cell-wall isolates were prepared from lyophilized whole cells as detailed before [18]. The isolated cell walls were digested into muropeptides with lysozyme and mutanolysin by using a modified version of the protocol described by others $[1,8]$. All manipulations were performed at neutral $\mathrm{pH}$.

\section{LC-MS/MS}

Liquid chromatography/MS and MS/MS were performed by using a PicoView PV-500 (New Objective, Woburn, MA) nanospray stage attached to either an LTQ-FT mass spectrometer or an LTQ-Orbitrap mass spectrometer (ThermoFisher, San Jose, CA).

Muropeptide samples were loaded into an uncoated 75 um i.d. fused-silica capillary column with a $15 \mu \mathrm{m}$ picofrit tip (New Objective, Woburn, MA), packed with C18 reverse-phase material $(3 \mu \mathrm{m}, 100 \AA$; Phenomenex, Torrance, CA). The column was eluted at a flow rate of $250 \mathrm{~nL} / \mathrm{min}$ for $10 \mathrm{~min}$ with $0.1 \%$ (vol/vol) formic acid in water and subsequently with a $60-$ min linear acetonitrile gradient $(0 \%-40 \%)$ with $0.1 \%$ formic acid. The samples, as they emerged from the column, were sprayed into an LTQ-FT mass spectrometer. Full mass spectra were recorded in the FT component of the instrument at 100,000 resolving power $($ at $\mathrm{m} / \mathrm{z}=400$ ).

Accurate mass product-ion spectra of muropeptides were acquired by introducing the samples by nanospray as they eluted from the LC to an LTQ-Orbitrap mass spectrometer. To obtain major components' production spectra (i.e., species in Scheme 2), cycles consisting of one full FT-scan mass spectrum and five ensuing data-dependent MS/MS scans acquired by the Orbitrap (with a normalized collision energy setting of 35\%) were repeated continuously throughout the elution with the following dynamic exclusion settings: repeat count, 3; repeat duration, $15 \mathrm{~s}$; exclusion duration, $30 \mathrm{~s}$. Sometimes, for minor components, an inclusion list containing the precursor ions' masses was applied.

\section{Solid-State NMR}

Experiments were performed using a four-frequency transmission-line probe [19] with a 14-mm long, 9-mm i.d. analytical coil, and a Chemagnetics/Varian magicangle spinning ceramic stator. Lyophilized samples were spun in Chemagnetics/Varian 7.5-mm o.d. Zirconia rotors at $5.0 \mathrm{kHz}$ under active control within $\pm 2 \mathrm{~Hz}$. Experiments were performed at room temperature with a Chemagnetics CMX-300 spectrometer operating at 
75.453 $\mathrm{MHz}$ for carbon. Radio frequency pulses were produced by 1-kW Kalmus, ENI, and American Microwave Technology power amplifiers, each under active control [20]; $\pi$ pulse lengths were $10 \mu$ s for ${ }^{15} \mathrm{~N}$. Cross-polarization transfer was at $50 \mathrm{kHz}$ for $2 \mathrm{~ms}$. Proton dipolar decoupling was at $105 \mathrm{kHz}$ during data acquisition. The data were taken from a cell-wall sample with a mass of $150 \mathrm{mg}$, isolated from a $4 \mathrm{~L}$ preparation.

\section{Results and Discussion}

\section{Muropeptide Identification}

Enterococcal peptidoglycan-repeat units (see Scheme 1) are interconnected by cross-linked D-aspartic acid or D-asparagine (D-Asx) bridges and via the polymerization of the disaccharides. We used $N$-acetylmuramidase enzymes to catalyze selectively the hydrolysis of the $\beta-1,4$ linkages between $N$-acetylmuramic acid and $N$ acetylglucosamine, leaving the cross-links intact. As expected, on the basis of previous studies $[8,9]$, most of the muropeptide peaks in the ion chromatogram (Figure 1) were variations of monomers, dimers, and a few trimers.

All of the assigned muropeptide species eluted with a retention time between 15 and $35 \mathrm{~min}$. The mass spectra, acquired so that accurate masses were taken for each scan of the ion chromatogram, serve as a means for evaluating muropeptide contributions. We considered first accurate-masses that are consistent with traditional muropeptide structures in a sample prepared with natural abundance isotopes only (no enrichment). These structural assignments were confirmed with MS/MS and stable-isotope labeling experiments. For example, an ion of $\mathrm{m} / \mathrm{z} 824.4$ corresponds to a species

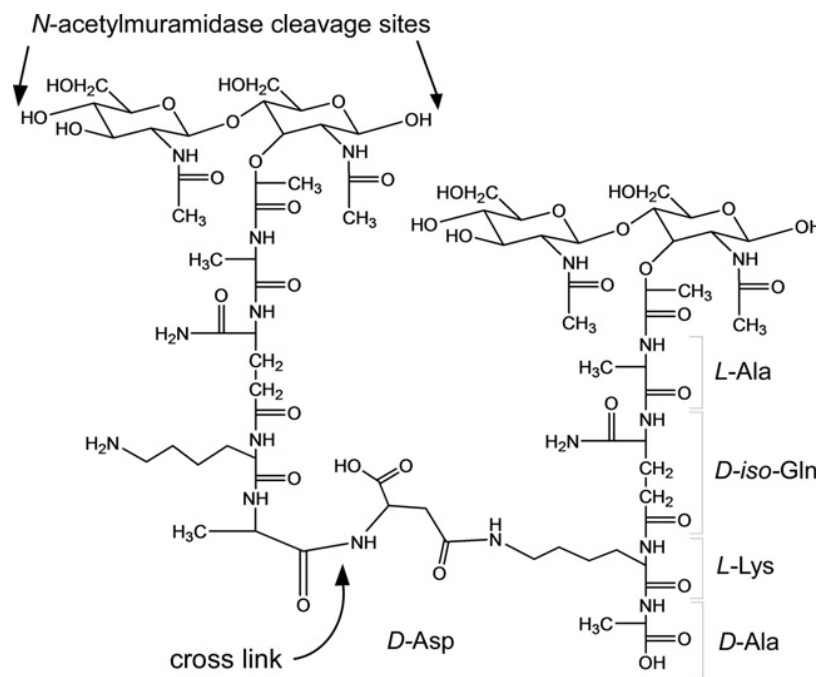

Scheme 1. Representative chemical structure of E. faecium peptidoglycan. The $\beta-1,4$ linkages between $N$-acetylmuramic acid and $\mathrm{N}$-acetylglucosamine are cleaved by $\mathrm{N}$-acetylmuramidase as indicated for the repeat unit on the left. The cross-links remain intact. D-iso-glutamine and D-aspartic acid may be substituted by either D-iso-glutamic acid or D-asparagine, respectively.

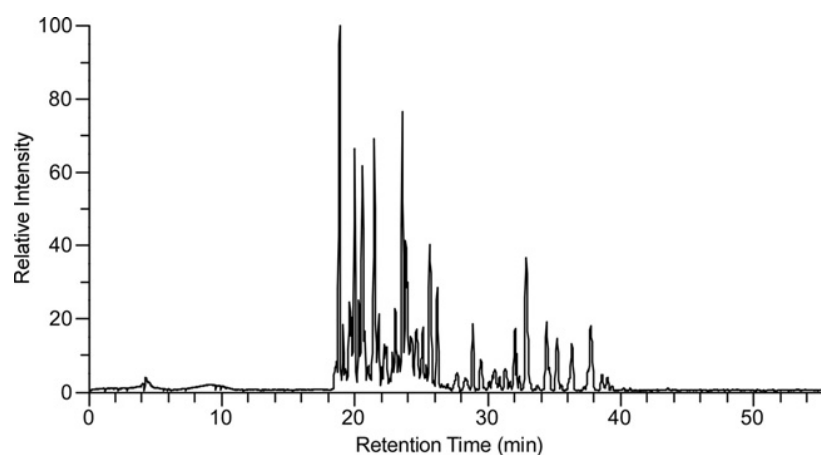

Figure 1. Total ion chromatogram for digested E. faecium muropeptides. The relative intensity is represented by total ion current as a function of retention time.

with a tripeptide stem without a bridge (see Scheme 2, Structure 2). When E. faecium is grown in ESM with double-labeled aspartic acid, this mass is unaffected. When the sample is grown with $\mathrm{L}-\left[1-{ }^{13} \mathrm{C}\right]$ lysine and $\mathrm{D}-\left[{ }^{15} \mathrm{~N}\right]$ alanine, the mass of the species increases by that consistent with the incorporation of one ${ }^{13} \mathrm{C}$.

Most of the other masses within 15-35 min retention time could be recognized as a variation of one of the traditional muropeptide structures. In fact, we were able to assign components represented by $\sim 90 \%$ of the peaks in the mass spectra as muropeptide structures and verify them by MS/MS and stable-isotope labeling experiments. It should be emphasized that in the isolation of the muropeptides, the peptidoglycan was not subjected to any acid or base treatments. Thus, we associate nearly all of the identified muropeptide structures as representative of enterococcal peptidoglycan.

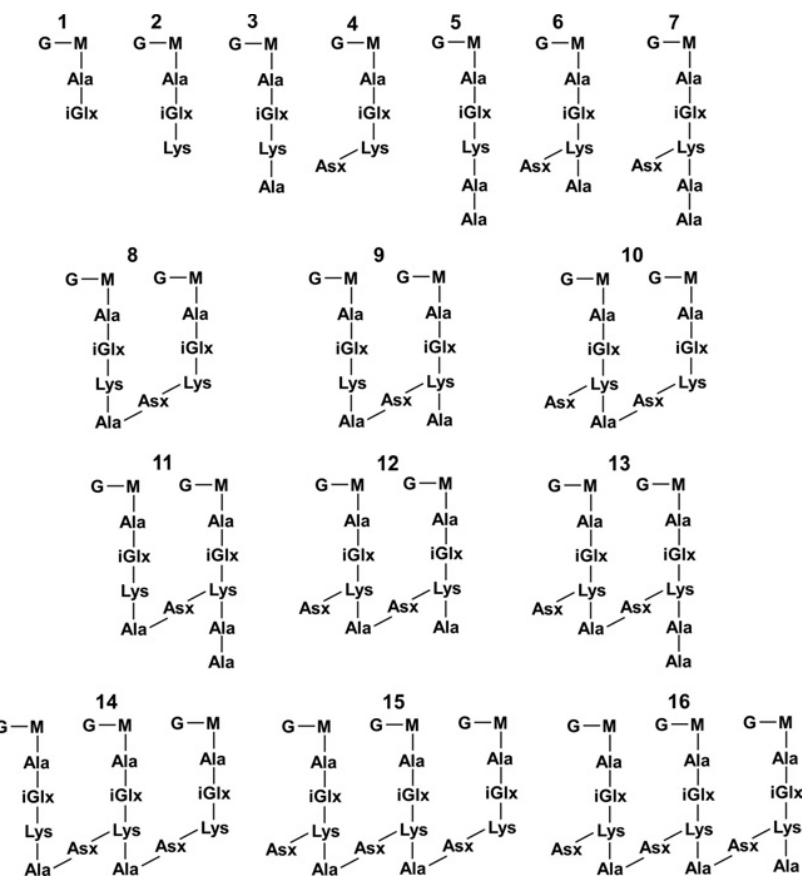

Scheme 2. Structures of assigned muropeptides. The relative proportion of each structure is shown in Table 1. 
The few exceptions found were not detected consistently in different sample preparations, and consequently ignored in the analysis.

\section{Structural Quantification}

The quantification of each muropeptide species was estimated using integrals of selected ion chromatograms. For species having structural isomers, the integral was taken as the sum over the entire selected ion chromatogram. Multiply charged species were also included in the integrals, and the distributions obtained by considering multiply charged ions were comparable to that of singly charged ions. The chromatographic separation has provided sufficient resolution such that the overlap of major and minor muropeptide components is minimal.

The monomers, dimers, and trimers represent $47 \%$, $43 \%$, and $8 \%$ of the muropeptides, respectively. The identified muropeptide structures (Scheme 2) and their relative abundances are listed in Table 1 . The number of peptidoglycan stems terminating in D-Ala-D-Ala is only $3 \%$. This quantification is reported as a percentage normalized to the total number of peptidoglycan stems. That is, dimer integrals are counted twice and trimer integrals are counted three times in determining the total number of peptidoglycan stems. This result is consistent with that determined in solid-state NMR studies of the same vancomycin-susceptible E. faecium organism, where the number of D-Ala-D-Ala terminating stems was found to be $7 \%$ in whole cells [17]. While peptidoglycan precursors and $N$-acetylglucosamine- $N$-acetyl-muramylpentapeptide-pyrophosphoryl-undecaprenol (lipid II) may contribute to the difference in measurements for the concentration of D-Ala-D-Ala stems, the determinations are still within experimental error (see Validity of MS Quantification: Comparison with Solid-State NMR Results section); we are unwilling to attribute any significance to the discrepancy.

\section{O-Acetylated Muropeptides}

We observed several structural variations of each identified muropeptide species, one of which is consistent with $\mathrm{O}$-acetylation of $\mathrm{N}$-acetylmuramic acid corresponding to a mass increase of 42.011 (Figure 2, second from bottom). O-acetylation of peptidoglycan has been well studied since it was first reported in 1958 [21, 22]. Thus far, all analyses demonstrate that $O$-acetylation only occurs at C6 of $\mathrm{N}$-acetylmuramic acid [23]. The product-ion spectra (MS/MS) of structures with and without $\mathrm{O}$-acetylation show the same fragment masses of $N$-acetylglucosamine and the peptide stem. On the other hand, the mass of $N$-acetylmuramic acid-peptide stem fragments increases by 42.011 for species with $\mathrm{O}$-acetylation. These observations provide strong support for the previous conclusions.

The ester bond of $O$-linked acetate is sensitive to mild alkaline hydrolysis [24]. As described above, the peptidoglycan was isolated at physiological $\mathrm{pH}$, leaving $\mathrm{O}$-acetylated peptidoglycan intact for MS experiments. Our results suggest that each muropeptide structure has a different likelihood of being O-acetylated (Table 2 ). We estimate that $5-15 \%$ of the total peptidoglycan is $\mathrm{O}$-acetylated on average. The extent of $\mathrm{O}$-acetylation is significantly lower than the $46-57 \%$ that was previously

Table 1. Distribution of structures in E. faecium peptidoglycan

\begin{tabular}{|c|c|c|c|c|}
\hline Species & Theoretical mass ${ }^{a}$ & Measured mass & Percentage $[\%]^{\mathrm{b}, \mathrm{c}}$ & Percentage $[\%]^{\mathrm{c}, \mathrm{d}}$ \\
\hline Monomers & & & 47 & 30 \\
\hline 1 & 695.2856 & 695.2855 & $<1$ & $<1$ \\
\hline 2 & 823.3805 & 823.3802 & 22 & 13 \\
\hline 3 & 894.4177 & 894.4174 & 5 & 3 \\
\hline 4 & 938.4075 & 938.4071 & 13 & 8 \\
\hline 5 & 965.4548 & 965.4555 & $<1$ & $<1$ \\
\hline 6 & 1009.4446 & 1009.4446 & 6 & 4 \\
\hline 7 & 1080.4817 & 1080.4825 & $<1$ & $<1$ \\
\hline Dimers & & & 43 & 51 \\
\hline 8 & 1814.8151 & 1814.8150 & 8 & 10 \\
\hline 9 & 1885.8522 & 1885.8510 & 3 & 4 \\
\hline 10 & 1929.8421 & 1929.8420 & 23 & 27 \\
\hline 11 & 1956.8894 & 1956.8899 & 2 & 2 \\
\hline 12 & 2000.8792 & 2000.8784 & 7 & 8 \\
\hline 13 & 2071.9163 & 2071.9158 & $<1$ & $<1$ \\
\hline Trimers & & & 8 & 15 \\
\hline 14 & 2806.2497 & 2806.2450 & 2 & 3 \\
\hline 15 & 2921.2767 & 2921.2767 & 5 & 10 \\
\hline 16 & 2992.3138 & 2992.3140 & 1 & 2 \\
\hline Tetramers & & & 2 & 4 \\
\hline
\end{tabular}

aTheoretical masses were calculated with Xcalibur 2.0.

${ }^{b}$ Percentages with respect to monomers, dimers, trimers, and tetramers.

'Both percentages include structural variations of the species (see Table 2). Under optimized digestion conditions for data from triplicate runs of four different sample preparations, each species contribution was within $\pm 3 \%$ of the values listed here.

dPercentages with respect to total number of peptidoglycan stems. 

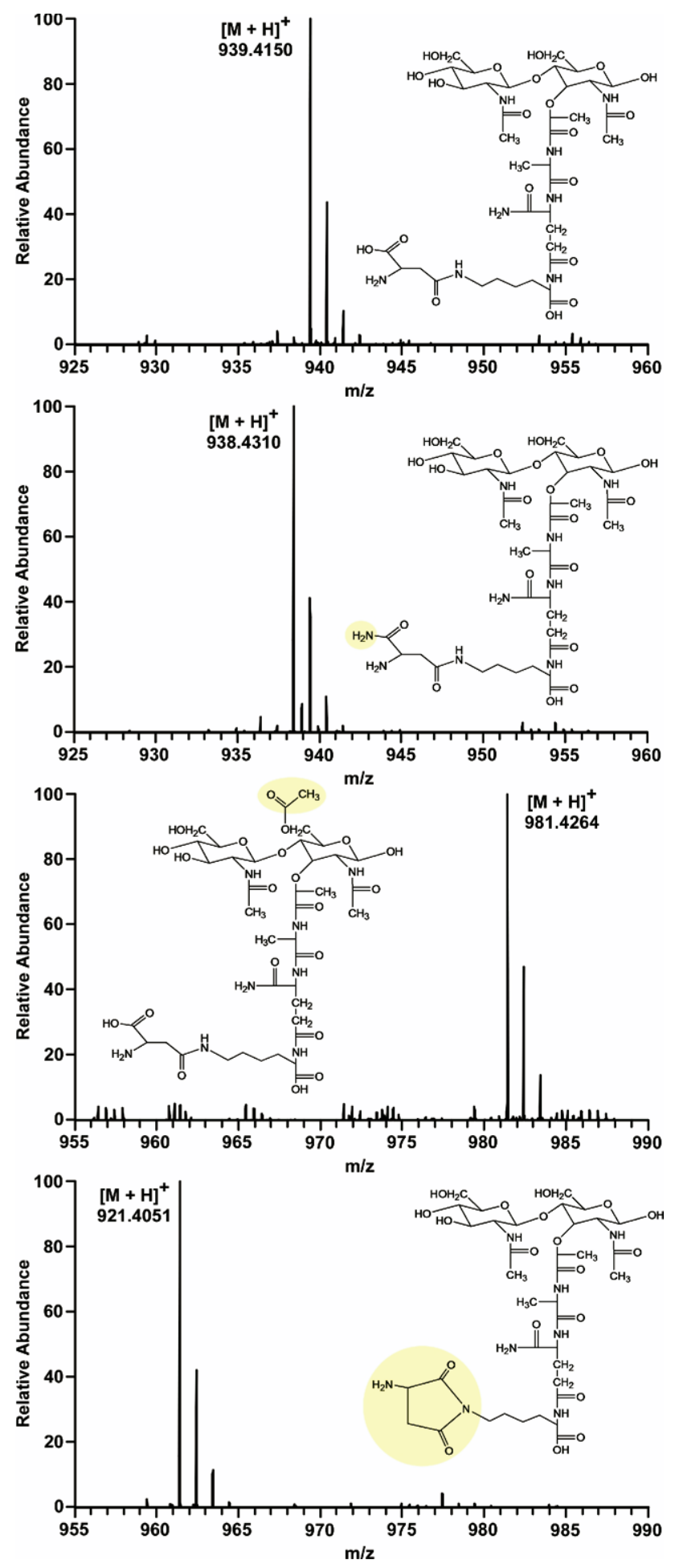

Figure 2. Mass spectra and assigned structures for variations of the muropeptide of $\mathrm{m} / \mathrm{z}=939.4150$. The most likely structure for an $m / z 939.4150$ ion (top) is that with a D-aspartic acid bridge. An $\mathrm{m} / \mathrm{z} 938.4310$ ion (second from top) corresponds to the muropeptide with double amidation, D-asparagine and D-iso-glutamine. A mass increase of 42.011 is consistent with $\mathrm{O}$-acetylation of $\mathrm{N}$ acetylmuramic acid (second from bottom). A mass decrease of 18.010 suggests the loss of water, and could be indicative of two types of cyclic imides (bottom). The structure shown is consistent with NMR data (see Figure 4). reported for E. faecium [25]. As was shown for other species [26-28], the extent of $O$-acetylation may vary in different enterococcal strains [25] and may even be correlated with vancomycin susceptibility in E. faecium.

In digesting the peptidoglycan, we used two $\mathrm{N}$ acetylmuramidase enzymes, mutanolysin and lysozyme. For many organisms, $\mathrm{O}$-acetylation renders peptidoglycan resistant to the activity of lysozyme [28], whereas mutanolysin isolated from Streptomyces globisporus is not affected by this modification [29]. Indeed, in the E. faecium preparations digested with only lysozyme, we observed no species with a mass increase consistent with $O$-acetylation for any muropeptide structures. Earlier studies showed large variations between different enterococcal strains in the lytic response of their cell walls to lysozyme [30, 31], indicating different degrees of $O$-acetylation [32]. Thus, it is not surprising that the extent of $O$-acetylation in the strain of E. faecium we investigated varies from that of a different strain reported before.

We also observed muropeptide structures that were $\mathrm{N}$-deacetylated, corresponding to a mass decrease of 42.011. The loss of an acetyl residue from $N$ acetylglucosamine or $\mathrm{N}$-acetylmuramic acid is infrequent in E. faecium, detected in less than $0.2 \%$ of the peptidoglycan stems. We, therefore, ignored these structures in determining the extent of $O$-acetylation as it is highly unlikely that significant numbers of muropeptides are simultaneously $\mathrm{O}$-acetylated and $\mathrm{N}$-deacetylated.

\section{Amidation of $D$-iso-Glu and $D$-Asp}

The occurrence of D-iso-glutamic acid residues in muropeptide components is hypothesized to affect crosslinking [33] and vancomycin resistance in Staphylococcus aureus [34]. Nonamidated peptidoglycan stems may be inefficient substrates for transpeptidase [35] and have higher affinity for vancomycin [34], thereby consuming the antibiotic in the cell wall before it can inhibit cell-wall biosynthesis at lipid II. E. faecium peptidoglycan has two potential sites for amidation, D-isoglutamine or D-iso-glutamic acid (D-iso-Glx) and D-Asx (see Scheme 1). Thus, for E. faecium peptidoglycan stems with bridges, there are three potential ions corresponding to double amidation, amidation/hydroxylation, and double hydroxylation. Because species with D-isoglutamine/D-aspartic acid and D-iso-glutamic acid/ D-asparagine have the same accurate mass, MS identification of each is difficult. There are, however, relatively few muropeptide structures without bridges that have a mass corresponding to hydroxylation. The singly charged muropeptide with an $\mathrm{m} / \mathrm{z}$ of 824.4 (see Scheme 2, Structure 2) does not have a bridge and contains D-iso-glutamine. The peak area for the ion of $\mathrm{m} / \mathrm{z}$ 825.4, representing an accurate mass increase of 0.984 , corresponding to hydroxylation, is relatively small compared with that of 824.4 (Figure 3a). This is consistent with there being very few D-iso-glutamic acid- 
Table 2. Distribution of selected monomers

\begin{tabular}{cccccc}
\hline Species & Acetylation [\%] & Cyclic Imide [\%] & Gln/Asn [\%] & Gln/Asp (Glu/Asn) [\%] & Glu/Asp [\%] \\
\hline \hline 2 & 19 & & & & \\
3 & 2 & 8 & 41 & 88 & 1 \\
4 & 11 & 3 & 16 & 73 & 1 \\
5 & 4 & 2 & 23 & 75 \\
6 & 3 & 2 & & \\
\hline
\end{tabular}

containing peptidoglycan stems, as was suggested for other enterococcal organisms [9]. Additionally, it is rare that muropeptides with bridges have a mass corresponding to double hydroxylation, supporting that few stems have D-iso-glutamic acid. Thus, we conclude that most muropeptide species with a mass increase corresponding to a single hydroxylation represent $\mathrm{D}-\mathrm{iso}$ glutamine/D-aspartic acid stems. Approximately 60\% of peptidoglycan bridges are $\mathrm{D}$-aspartic acid, and $2 \%$ of all peptidoglycan stems contain D-iso-glutamic acid (Table 2).

\section{Structural Bridge Isomers}

Selected ion chromatograms of muropeptides with bridges show two peaks representing components with different retention times, suggesting that these muropeptides exist as two structural isomers associated with unique bridge conformations (Figure 3). The deamidation of $\mathrm{D}$-asparagine is known to produce a mixture of $\alpha$ - and $\beta$-D-aspartic acid residues [36-38]. Recently, however, it was suggested that D-asparagine bridges in E. faecium exclusively originate from the amidation of D-aspartic acid after its incorporation into the peptidoglycan [39]. Further, the incorporation is thought to proceed specifically by way of a $\beta$-aspartylphosphate intermediate [40, 41], making $\alpha$ - and $\beta$ bridge isomers unlikely. Considering that $\beta$-D-aspartic acid is incorporated into peptidoglycan precursors, and that the 1 and 4 aspartyl-carbons are similar, we cannot

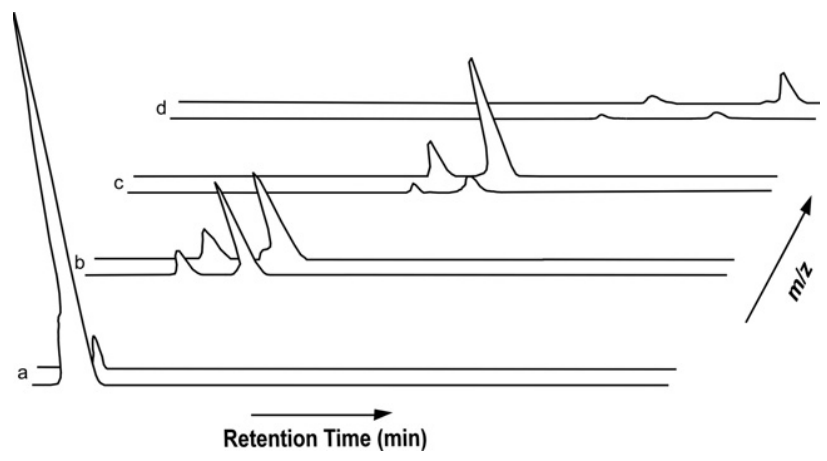

Figure 3. Selected ion chromatograms for singly charged species with $\mathrm{m} / \mathrm{z}$ of (a) 824.4 and 825.4; (b) 938.4 and 939.4; (c) 1009.5 and 1010.5; and (d) 1080.5 and 1081.5. The relative intensities of 1080.5 and 1081.5 are small, consistent with there being few D-Ala-D-Ala stems. Muropeptides with bridges elute as two distinct peaks with different retention times in the chromatogram. The selected ion chromatogram for $\mathrm{m} / \mathrm{z} 824.4$ shows only one peak. rule out the possibility that either carbon could form a peptide bond with the 6-N-lysine, thereby creating two unique bridge conformations.

\section{Cyclic Imides}

Interestingly, the muropeptide monomers we assigned to have aspartic acid bridges show a relatively small amount of species with an accurate mass decrease of 18.010 consistent with the loss of water (Figure 2, bottom). MS/MS experiments indicate that the water loss is not from the disaccharides or the first amino acid of the peptide stem, L-alanine. Moreover, tripeptide monomers with bridges (see Scheme 2, Structure 4) also undergo water loss before mass spectrometry analysis. We hypothesize that the water loss results in the formation of a cyclic imide structure for which there are at least two possibilities. Aspartic acid could form a succinimide with 6-N-lysine (see Figure 2, bottom) or undergo cyclization with $\mathrm{N}$-D-iso-glutamine.

Using the current mass spectrometric data, we are unable to differentiate between the two proposed varieties of cyclic imides. Monomeric muropeptides containing D-iso-glutamine and D-asparagine do not show mass decreases consistent with water loss, probably because there is no free hydroxyl to be protonated and become a leaving group. If water loss were to occur from stems containing both $\mathrm{D}$-iso-glutamic acid and D-aspartic acid, it would suggest that the succinimide is the favored structure. Unfortunately, the infrequency of D-iso-glutamic acid containing muropeptide stems makes this analysis difficult. It should be noted that some structures with a missing water molecule, such as that with an $\mathrm{m} / \mathrm{z}$ of 939.4 , produce two peaks with different retention times in the ion chromatogram. This may be representative of both types of cyclic imide structures or a consequence of there being two bridge orientations as discussed above.

Solid-state NMR experiments performed on intact cell-wall isolates specifically labeled with $\mathrm{L}-\left[6^{-15} \mathrm{~N}\right] \mathrm{l}-$ ysine provide insight in the identification. An ${ }^{15} \mathrm{~N}$ cross-polarization magic-angle spinning (CPMAS) echo spectrum is consistent with a succinimide structure (Figure 4). The peak at $5 \mathrm{ppm}$ is due to lysyl amines and the peak at $95 \mathrm{ppm}$ is for lysyl amides. We suggest that the small peak at $151 \mathrm{ppm}$ is from lysyl succinimides, representing a small percentage of peptidoglycan bridges. Although succinimides were proposed as in- 


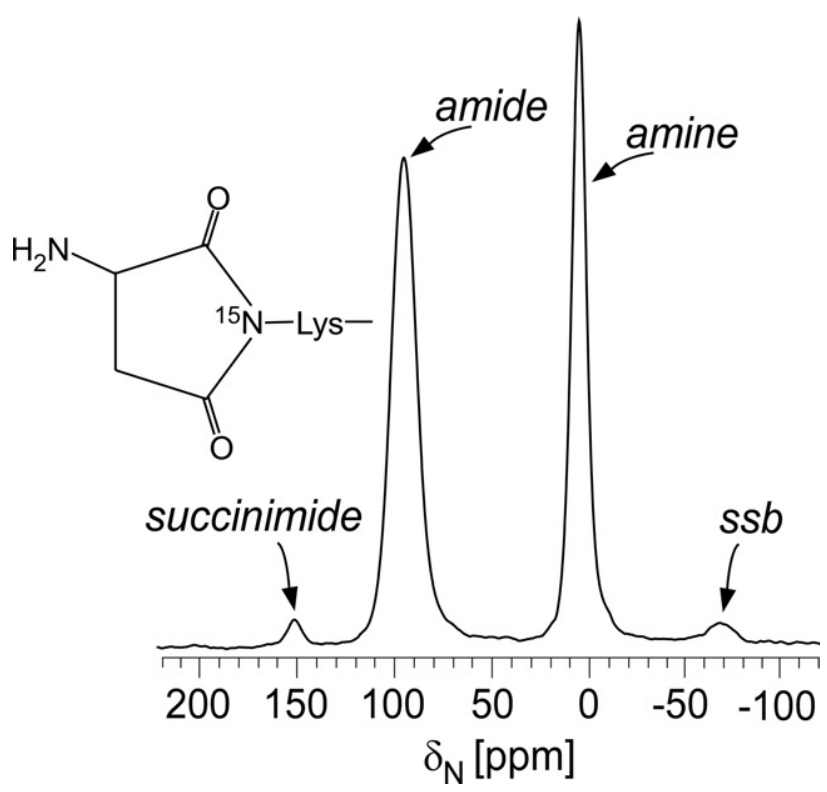

Figure 4. ${ }^{15} \mathrm{~N}$ CPMAS echo-spectrum of E. faecium cell-wall

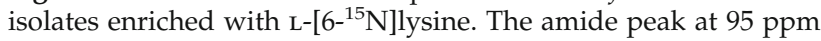
occurs when lysine has a D-Asx bridge attached, and the amine peak at 5 ppm occurs when lysine does not have an attached bridge. We assign the $151 \mathrm{ppm}$ peak to lysyl succinimides.

termediates [36], to our knowledge, this is the first detection of such structures in E. faecium peptidoglycan.

\section{Muropeptides Without Asx Bridges}

The solid-state NMR ${ }^{15} \mathrm{~N}$ CPMAS echo spectrum of $E$. faecium cell-wall isolates shows, from the ratio of the amine to amide peak, that a significant percentage of peptidoglycan stems do not have bridges [17]. This result contrasts with those from previous mass spectrometric studies in which it was concluded that over $95 \%$ of the enterococcal peptidoglycan contains bridges [8]. Results from our MS experiments are consistent with solid-state NMR data, showing nearly $30 \%$ of peptidoglycan stems do not have bridges. The significant number of muropeptide stems without bridges is shown by the relative intensity of $\mathrm{m} / \mathrm{z} 824.4$ (amidated species 2 of Scheme 2) compared with other species (see Figure 3). The discrepancy in bridge-links between our data and those given in previous MS studies may be related to the specific strain of E. faecium investigated, or it may result from excessive mutanolysin digestion of muropeptides. The cell walls we digested with only lysozyme showed significant proportions of lysyl amine structures, but no structures consistent with $O$-acetylation. In cell walls digested with high concentrations of mutanolysin and lysozyme for $24 \mathrm{~h}$, lysyl amine muropeptides decreased, representing less than $5 \%$ of the peptidoglycan. Additionally, the ion chromatogram profile changed. For example, the ratio of structures with an $\mathrm{m} / \mathrm{z}$ of 824.4 to that of 825.4 (hydroxylated species 2 of Scheme 2) changed by a factor of six times, indicating that high concentrations of mutanolysin may further degrade some muropeptides after having cleaved the $\beta-1,4$ glycan linkages. To optimize the digestion, the concentration and digestion times were reduced such that the treatment produced significant proportions of lysyl amine muropeptides consistent with solid-state NMR measurements made on cell-wall isolates before digestion while still solubilizing a substantial amount of peptidoglycan and cleaving $\mathrm{O}$-acetyl-substituted oligomers.

\section{Validity of MS Quantification: Comparison with Solid-State NMR Results}

The MS quantifications in this report are based on integrals obtained from ion chromatograms, and given that there are likely to be differences in ionization efficiencies between muropeptide structures and potential digestion sampling problems, we view them as approximations. Nonetheless, the investigation has revealed several new structural details of $E$. faecium peptidoglycan, the relative proportion of each motif, and the types of stems on which they occur. As a method to judge the quantitative integrity of the MS results, the percentage of total peptidoglycan stems cross-linked can be calculated and compared with that directly measured by solid-state NMR. Cross-linking is equal to: (dimers $+2 \cdot$ trimer $+3 \cdot$ tetramers $) /($ monomers $+2 \cdot$ dimers +3 . trimers +4 - tetramers). From the MS data, $\sim 40 \%$ of E. faecium peptidoglycan is cross-linked. This result is in good agreement with the $47 \%$ obtained from solid-state NMR [17].

\section{Glycan Chain Lengths}

The absence of cross-linked chains longer than tetramers contrasts with the peptidoglycan from Staphylococcus aureus, another gram-positive pathogen, where cross-linked chains greater than 9-mers were observed after $\mathrm{N}$-acetylmuramidase digestion [42]. In comparison to $S$. aureus, we propose that $E$. faecium compensates for the lack of peptidoglycan integrity resulting from short cross-linked fragments by extending the length of the glycan chains. No data have been reported for E. faecium glycan chain length, but we anticipate that it will be much longer than in S. aureus, where the average was reported to be six disaccharides [43]. Average glycan lengths as long as 140 disaccharides have been reported in some B. subtilis species [44].

\section{Template Model for Cell-Wall Biosynthesis}

Both MS and solid-state NMR results show that the number of peptidoglycan stems terminating in D-AlaD-Ala is small despite this organism's susceptibility to vancomycin. Nevertheless, these binding sites are crucial to cell-wall biosynthesis, and their small number is fundamentally consistent with the template model proposed for $S$. aureus $[6,7]$ and recently 


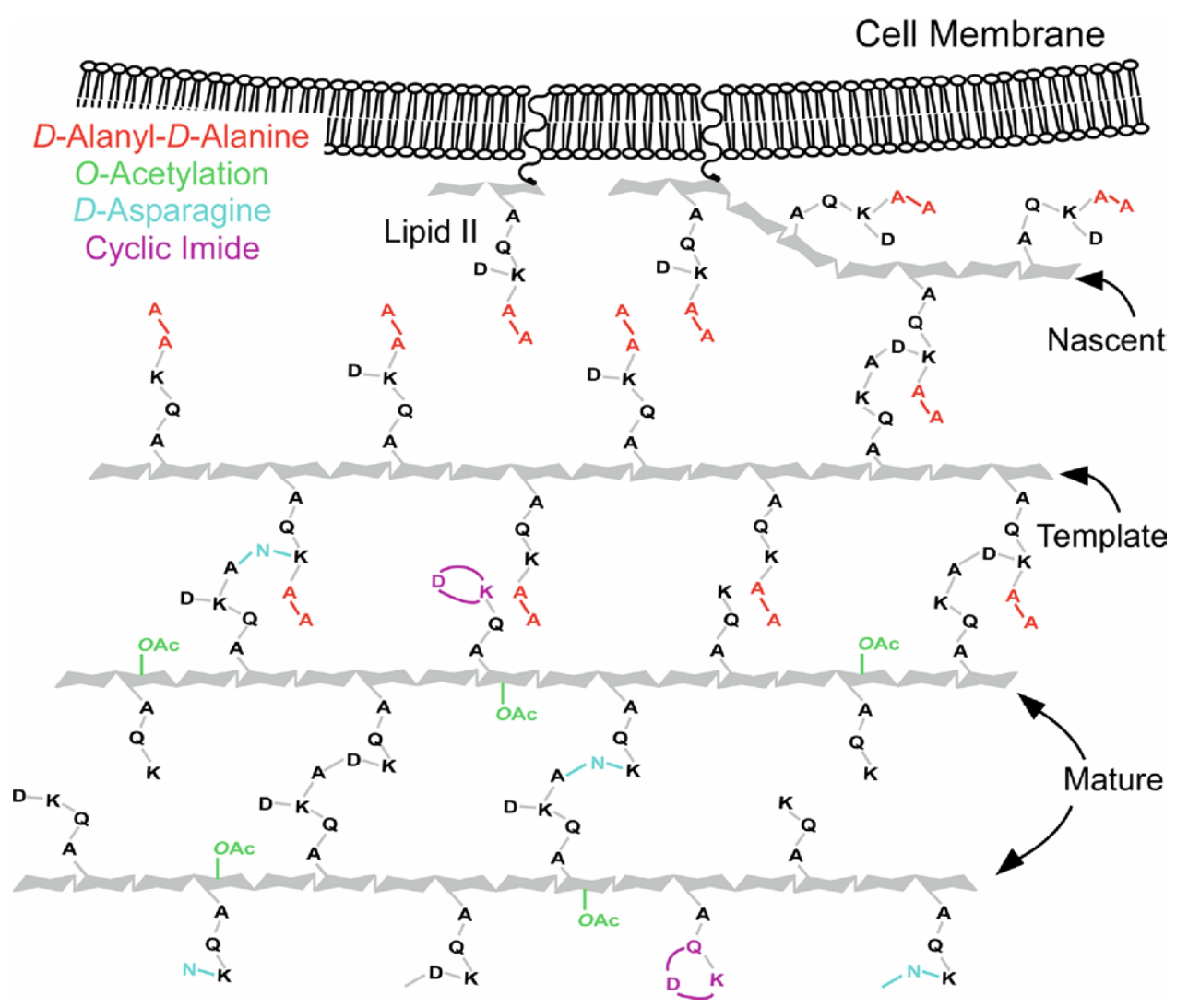

Figure 5. Two-dimensional schematic representation of the template model of peptidoglycan biosynthesis for E. faecium. Chain extension occurs from right to left and is synchronized with cross-linking at lipid II. The few peptidoglycan stems terminating in D-Ala-D-Ala (red) occur in nascent and template peptidoglycan where they are cross-linked. We suspect that L,D-carboxypeptidase acts on stems in mature peptidoglycan that are not cross linked. We propose that other cell-wall editing enzymatic processes occur in mature peptidoglycan as well, creating muropeptides with $\mathrm{O}$-acetylation (green), D-asparagine (blue), and cyclic imides (purple).

extended to E. faecium peptidoglycan assembly [17]. In this single-strand addition model, the orientation of nascent peptidoglycan is signaled by the stem conformation of the template, the last completed glycan chain (Figure 5). For the template to form cross-links with the extending nascent peptidoglycan, it must have D-Ala-DAla stems. These few stems represent the $3 \%$ of all D-Ala-D-Ala peptidoglycan observed in MS experiments. Modification of the few D-Ala-D-Ala stems in nascent and template peptidoglycan prevents the binding of vancomycin and its ensuing inhibition of the enzymatic processes crucial for cell-wall assembly. We suspect that there is an active L,D-carboxypeptidase that cleaves D-Ala-D-Ala stems that are not cross-linked in mature peptidoglycan. It was suggested that L,D-carboxypeptidase activity is involved in peptidoglycan maturation for other organisms [45-48]. In addition to L,D-carboxypeptidase activity, we propose that other enzymatic modifications also occur in mature peptidoglycan. Examples include $O$-acetylation, amidation of aspartic acid, and formation of cyclic imides, which occur less frequently on muropeptides associated with nascent and template peptidoglycan, namely those with D-Ala-D-Ala stems (see Table 2). The results suggest that significant processing occurs in mature peptidoglycan where several enzymes are active in editing cell-wall structure. Modified mature peptidoglycan may play an important role in biosynthetic regulation and prove important in virulence, allowing bacterial infections to evade host-immune system response.

\section{Conclusions}

Mass spectrometry, particularly in LC/MS mode in which accurate masses and product-ion spectra are acquired, can provide new structural detail pertaining to variations of peptidoglycan stems. These results may prove important in understanding glycopeptideantibiotic binding. One source of success of our bottom-up approach is the gentle treatments for digestion under neutral $\mathrm{pH}$ that allow the fine structure of the peptidoglycan to be preserved. Using this gentle digestion, we implemented an on-line LC/MS analysis that gives results suitable for comparison with those from solidstate NMR, a method with excellent quantitative sensitivity and reliability. The comparison verifies that, despite being susceptible to vancomycin, vancomycinsusceptible Enterococcus faecium have very few D-Ala-D- 
Ala binding sites. The finding is consistent with a template model for cell-wall biosynthesis and suggests that $\mathrm{L}, \mathrm{D}$-carboxypeptidase activity exists in E. faecium peptidoglycan. The importance of infrequently occurring peptidoglycan structural motifs, such as the binding site of vancomycin, underscores the need for characterizing fine peptidoglycan structural details, as was accomplished by this research.

\section{Acknowledgments}

The authors acknowledge support for this research by the National Centers for Research Resources of National Institutes of Health (grant no. P41RR000954) and the National Institutes of Health (grant no. EB002058).

\section{References}

1. Uttley, A. H.; Collins, C. H.; Naidoo, J.; George, R. C. VancomycinResistant Enterococci. Lancet 1988, 1, 57-58.

2. Kak, V. A. C.; Joseph, W. Acquired antibiotic resistances in enterococci; ASM Press: Washington, DC, 2002; 359-360.

3. Mainardi, J. L.; Villet, R.; Bugg, T. D.; Mayer, C.; Arthur, M. Evolution of Peptidoglycan Biosynthesis Under the Selective Pressure of Antibiotics in Gram-Positive Bacteria. FEMS Microbiol. Rev. 2008, 32, 386-408.

4. Courvalin, P. Vancomycin Resistance in Gram-Positive Cocci. Clin. Infect. Dis. 2006, 42(Suppl. 1), S25-S34.

5. Bugg, T. D.; Wright, G. D.; Dutka-Malen, S.; Arthur, M.; Courvalin, P.; Walsh, C. T. Molecular Basis for Vancomycin Resistance in Enterococcus faecium BM4147: Biosynthesis of a Depsipeptide Peptidoglycan Precursor by Vancomycin Resistance Proteins VanH and VanA. Biochemistry 1991, 30, 10408-10415.

6. Kim, S. J.; Cegelski, L.; Stueber, D.; Singh, M.; Dietrich, E.; Tanaka, K. S. E.; Parr, T. R.; Far, A. R.; Schaefer, J. Mode of Action of Oritavancin in Staphylococcus aureus by Solid-State NMR. J. Mol. Biol. 2007, 377, 281-293.

7. Kim, S. J.; Matsuoka, S.; Patti, G. J.; Schaefer, J. Vancomycin Derivative with Damaged $D$-Ala-D-Ala binding cleft binds to cross-linked peptidoglycan in the cell wall of Staphylococcus aureus. Biochemistry 2008, 47, 3822-3831.

8. Billot-Klein, D.; Shlaes, D.; Bryant, D.; Bell, D.; van Heijenoort, J.; Gutmann, L. Peptidoglycan Structure of Enterococcus faecium Expressing Vancomycin Resistance of the VanB type. Biochem. J. 1996, 313(Pt 3), 711-715.

9. de Jonge, B. L.; Gage, D.; Handwerger, S. Peptidoglycan Composition of Vancomycin-Resistant Enterococcus faecium. Microb. Drug Resist. 1996, 2, 225-229.

10. Singh, P. D.; Johnson, J. H. Muraceins-Muramyl Peptides Produced by Nocardia orientalis as angiotensin-converting enzyme inhibitors. II. Isolation and Structure Determination. J. Antibiot. (Tokyo) 1984, 37, 336-343.

11. Pittenauer, E.; S. E. R., Allmaier, G.; Pfanzagl, B.; Loffelhardt, W.; Quintela, C.; de Pedro, M. A.; Stanek, W. Structural Characterization of the Cyanelle Peptidoglycan of Cyanophora paradoxa by Californium-252 Plasma Desorption Mass Spectrometry and Fast Atom Bombardment/ Tandem Mass Spectrometry. Biol. Mass Spectrom. 1993, 22, 524-536.

12. Pfanzagl, B.; Zenker, A.; Pittenauer, E.; Allmaier, G.; Martinez-Torrecuadrada, J.; Schmid, E. R.; De Pedro, M. A.; Loffelhardt, W. Primary Structure of Cyanelle Peptidoglycan of Cyanophora paradoxa: A Prokaryotic Cell Wall as Part of an Organelle Envelope. J. Bacteriol. 1996, 178, 332-339.

13. Zenker, A. P. B.; Loffelhardt, W.; Allmaier, G. Negative and Positive Ion Matrix-Assisted Laser Desorption Ionization Mass Spectrometry of Peptidoglycan Fragments After Size Fractionation and Reversed-Phase HighPerformance Liquid Chromatography. J. Microbiol. Methods 1998, 32, 237-246.

14. Bacher, G.; Korner, R.; Atrih, A.; Foster, S. J.; Roepstorff, P.; Allmaier, G. Negative and Positive Ion Matrix-Assisted Laser Desorption/Ionization Time-of-Flight Mass Spectrometry and Positive Ion Nano-Electrospray Ionization Quadrupole Ion Trap Mass Spectrometry of Peptidoglycan Fragments Isolated from Various Bacillus species. J. Mass Spectrom. 2001, 36, 124-139.

15. Glauner, B. Separation and Quantification of Muropeptides with HighPerformance Liquid Chromatography. Anal. Biochem. 1988, 172, 451-64.

16. Glauner, B.; Holtje, J. V.; Schwarz, U. The Composition of the Murein of Escherichia coli. J. Biol. Chem. 1988, 263, 10088-10095.

17. Patti, G. J., Kim, S. J., Schaefer, J. Characterization of the Peptidoglycan of Vancomycin-Susceptible Enterococcus faecium. Biochemistry; doi:10.1021/bi8008032

18. Tong, G.; Pan, Y.; Dong, H.; Pryor, R.; Wilson, G. E.; Schaefer, J. Structure and Dynamics of Pentaglycyl Bridges in the Cell Walls of Staphylococcus aureus by ${ }^{13} \mathrm{C}-{ }^{15} \mathrm{~N}$ REDOR NMR. Biochemistry 1997, 36, 9859-9866.

19. Schaefer, J.; McKay, R. A. Patent, U. S., 1999, 5,861,748.
20. Guillion, T. Schaefer J. Detection of Weak Heteronuclear Dipolar Coupling by Rotational-Echo Double-Resonance Nuclear Magnetic Resonance. Adv. Magn. Reson. 1989, 13, 57-83.

21. Abrams, A. O-Acetyl Groups in the Cell Wall of Streptococcus faecalis. J. Biol. Chem. 1958, 230, 949-959.

22. Brumfitt, W.; Wardlaw, A. C.; Park, J. T. Development of LysozymeResistance in Micrococcus lysodiekticus and Its Association with an Increased O-Acetyl Content of the Cell Wall. Nature 1958, 181, 1783-1784.

23. Vollmer, W. Structural Variation in the Glycan Strands of Bacterial Peptidoglycan. FEMS Microbiol. Rev. 2008, 32, 287-306.

24. Bera, A.; Herbert, S.; Jakob, A.; Vollmer, W.; Gotz, F. Why are Pathogenic Staphylococci so Lysozyme Resistant? The Peptidoglycan O-Acetyltransferase OatA is the Major Determinant for Lysozyme Resistance of Staphylococcus aureus. Mol. Microbiol. 2005, 55, 778-787.

25. Pfeffer, J. M.; Strating, H.; Weadge, J. T.; Clarke, A. J. Peptidoglycan. O Acetylation and Autolysin Profile of Enterococcus faecalis in the Viable but Nonculturable state. J. Bacteriol. 2006, 188, 902-908.

26. Rosenthal, R. S.; Folkening, W. J.; Miller, D. R.; Swim, S. C. Resistance of O-Acetylated Gonococcal Peptidoglycan to Human PeptidoglycanDegrading Enzymes. Infect. Immun. 1983, 40, 903-911.

27. Swim, S. C.; Gfell, M. A.; Wilde, C. E. III; Rosenthal, R. S. Strain Distribution in Extents of Lysozyme Resistance and O-Acetylation of Gonococcal Peptidoglycan Determined by High-Performance Liquid Chromatography. Infect. Immun. 1983, 42, 446-452.

28. Clarke, A. J.; Dupont, C. O-Acetylated Peptidoglycan: Its Occurrence, Pathobiological Significance, and Biosynthesis. Can. J. Microbiol. 1992, $38,85-91$.

29. Hamada, S.; Torii, M.; Kotani, S.; Masuda, N.; Ooshima, T.; Yokogawa, K.; Kawata, S. Lysis of Streptococcus mutans cells with Mutanolysin, a Lytic Enzyme Prepared from a Culture Liquor of Streptomyces globisporus 1829. Arch. Oral. Biol. 1978, 23, 543-549.

30. Chesbro, W. R. Lysozymes and the Production of Osmotic Fragility in Enterococci. Can. J. Microbiol. 1961, 7, 952-955.

31. Toennies, G.; Iszard, L.; Rogers, N. B.; Shockman, G. D. Cell Multiplication Studied with an Electronic Particle Counter. J. Bacteriol. 1961, 82, 857-866.

32. Metcalf, R. H.; Deibel, R. H. Growth of Streptococcus faecium in the Presence of Lysozyme. Infect. Immun. 1972, 6, 178-183.

33. Stranden, A. M.; Roos, M.; Berger-Bachi, B. Glutamine Synthetase and Heteroresistance in Methicillin-Resistant Staphylococcus aureus. Microb. Drug Resist. 1996, 2, 201-207.

34. Hanaki, H.; Labischinski, H. Inaba, $\mathrm{Y}$; Kondo, N. Murakami, H. Hiramatsu, K. Increase in Glutamine Nonamidated Muropeptides in the Peptidoglycan of Vancomycin-Resistant Staphylococcus aureus strain Mu50. J. Antimicrob. Chemother. 1998, 42, 315-320.

35. Nakel, M.; Ghuysen, J. M.; Kandler, O. Wall Peptidoglycan in Aerococcus viridans strains 201 Evans and ATCC 11563 and in Gaffkya homari strain ATCC 10400. Biochemistry 1971, 10, 2170-2175.

36. Ghuysen, J. M. Use of Bbacteriolytic Enzymes in Determination of Wall Structure and Their Role in Cell Metabolism. Bacteriol. Rev. 1968, 32, $425-464$.

37. Ikawa, M. The Configuration of Aspartic Acid in Cell Walls of Lactic Acid Bacteria and Factors Affecting the Racemization of Aspartic Acid. Biochemistry 1964, 3, 594-597.

38. Suginaka, H.; Kotani, S.; Kato, K.; Kashiba, S.; Amano, T. Action of a Staphylolytic Enzyme (ALE) of a Strain of Staphylococcus epidermidis. Biken's J. 1968, 11, 13-24.

39. Bellais, S.; Arthur, M.; Dubost, L.; Hugonnet, J. E.; Gutmann, L.; van Heijenoort, J.; Legrand, R.; Brouard, J. P.; Rice, L.; Mainardi, J. L. ASTM, the D-Aspartate Ligase Responsible for the Addition of D-Aspartic Acid onto the Peptidoglycan Precursor of Enterococcus faecium. J. Biol. Chem. 2006, 281, 11586-11594.

40. Staudenbauer, W.; Strominger, J. L. Activation of D-Aspartic Acid for Incorporation into Peptidoglycan. J. Biol. Chem. 1972, 247, 5095-5102.

41. Staudenbauer, W.; Willoughby, E.; Strominger, J. L. Further studies of the D-Aspartic Acid-Activating Enzyme of Streptococcus faecalis and Its Attachment to the Membrane. J. Biol. Chem. 1972, 247, 5289-5296.

42. de Jonge, B. L.; Chang, Y. S.; Gage, D.; Tomasz, A. Peptidoglycan Composition of a Highly Methicillin-Resistant Staphylococcus aureus strain. The Role of Penicillin Binding Protein 2A. J. Biol. Chem. 1992, 267, 11248-11254.

43. Boneca, I. G.; Huang, Z. H.; Gage, D. A.; Tomasz, A. Characterization of Staphylococcus aureus Cell Wall Glycan Strands, Evidence for a New $\beta$-N-Acetylglucosaminidase Activity. J. Biol. Chem. 2000, 275, 9910-9918.

44. Archibald, A. R.; Hancock, I. C.; Harwood, C. R. Cell wall structure, synthesis, and turnover; ASM Press: Washington, DC; 1993.

45. Courtin, P.; Miranda, G.; Guillot, A.; Wessner, F.; Mezange, C.; Domakova, E.; Kulakauskas, S.; Chapot-Chartier, M. P. Peptidoglycan Structure Analysis of Lactococcus lactis Reveals the Presence of an L,DCarboxypeptidase Involved in Peptidoglycan Maturation. J. Bacteriol. 2006, 188, 5293-5298.

46. Korza, H. J.; Bochtler, M. Pseudomonas aeruginosa LD-Carboxypeptidase, a Serine Peptidase with a Ser-His-Glu Triad and a Nucleophilic Elbow. J. Biol. Chem. 2005, 280, 40802-40812.

47. Templin, M. F.; Ursinus, A.; Holtje, J. V. A Defect in Cell Wall Recycling Triggers Autolysis During Stationary Growth Phase of Escherichia coli. EMBO J. 1999, 18, 4108-4117.

48. Ursinus, A.; Steinhaus, H.; Holtje, J. V. Purification of a Nocardicin A-Sensitive $L D$-carboxypeptidase from Escherichia coli by affinity chromatography. J. Bacteriol. 1992, 174, 441-446. 